\title{
PAUTAS DE EVALUACION DE LAS HABILIDADES MOTRICES BÁSICAS DE BOTEAR UN BALON, RODAR HACIA ADELANTE Y HACER RODAR UN BALON EN NIÑOS Y NIÑAS DE 6 Y 7 AÑOS DE EDAD
}

\section{GUIDELINES EVALUATION OF BASIC MOTOR SKILLS BODY ROLLING, DRIBBLING AND BALL ROLLING CHILDREN 6 AND 7 YEARS OLD}

\author{
Gamboa Jimenez, Rodrigo ${ }^{1}$; Acevedo Gallardo, Constanza ${ }^{1}$; Canala-Echeverria Tobar, Stefania ${ }^{1}$; Olivares \\ Villanueva, Ivon ${ }^{1}$; Valenzuela Montana, Katherine ${ }^{1}$; Jiménez Alvarado, Gladys ${ }^{1}$ \& Cacciuttolo Juárez, \\ Carola $^{2}$.
}

${ }^{1}$ Laboratorio Motricidad y Educación, Escuela de Educación Física, Pontificia Universidad Católica de Valparaíso.

${ }^{2}$ Carrera de Educación Parvularia, Universidad de Valparaíso.

GAMBOA J.R., ACEVEDO G.C., CANALA-ECHEVERRIA T.S., OLIVARES V.I., VALENZUELA M.K., JIMENEZ A.G. \& CACCIUTTOLO J.C. Pautas de Evaluación de las Habilidades Motrices Básicas de botear un balón, rodar hacia adelante y hacer rodar un balón en niños y niñas de 6 y 7 años de edad. Mot.Hum. 13(2) : 74-83 ; 2012.

\section{RESUMEN}

En el presente artículo, y como resultado del proceso investigativo, se pone al servicio de los docentes de la educación física un conjunto de pautas de evaluación de la calidad de ejecución de las habilidades motrices básicas de botear un balón con mano dominante, rodar hacia adelante y hacer rodar un balón con mano dominante, elaboradas conforme al patrón maduro de cada una de ellas. Para su validación, se obtiene la validez de contenido a través del juicio de expertos en la temática; en relación a la claridad, coherencia y pertinencia de los diferentes elementos que conforman cada una de las pautas; y se obtiene el grado de fiabilidad de los instrumentos evaluativos en una muestra de 100 niños y niñas de 6 y 7 años de edad de un establecimiento educativo de la ciudad de Quilpué. Lo anterior, por medio de la determinación del grado la confiabilidad, consistencia y objetividad interobservador. Para el procesamiento de la información, en el juicio de expertos, se aplicó el coeficiente de correlación de Spearman para obtener el grado de concordancia de dichos jueces; y para la fiabilidad, se aplican los índices KAPPA y ANOVA y Test de Comparaciones Múltiples de Tukey (confiabilidad, consistencia y objetividad interobservador). En relación a los resultados, se han obtenido altos grados de concordancia en todos los análisis realizados.

Palabras Clave: Habilidades motrices básicas, Pautas de evaluación, Patrón maduro de la habilidad.

\begin{abstract}
In the present article, and as result of the process investigative, there puts to the service of the teachers of the physical education a set of guidelines of evaluation of the quality of execution of the motive basic skills of dribbling on a ball with dominant hand, to roll ahead and to make roll a ball with dominant hand, elaborated in conformity with the mature boss of each one of them. For his validation, the validity of content is obtained across the experts' judgment in the subject matter; in relation to the clarity, coherence and relevancy of the different elements that agree each of the guidelines; and there is obtained the degree of reliability of the instruments evaluative in a sample of 100 children 6 and 7 year old of age of Quilpué's city, I become a member of association educations. The previous thing, by means of the determination of the degree the reliability, consistency and objectivity interobserver. For the processing of the information, in the experts' judgment, I apply to him the coefficient of Spearman's correlation to obtain the degree of conformity of the above mentioned judges; and for the reliability, there are applied the indexes KAPPA and ANOVA and Test of Tukey's Multiple Comparisons (reliability, consistency and objectivity interobserver). In relation to the results, high degrees of conformity have been obtained in all the realized analyses.
\end{abstract}

Keywords: Motors basic Skills, Guidelines of evaluation, mature boss of the skill. 
GAMBOA J.R., ACEVEDO G.C., CANALA-ECHEVERRIA T.S., OLIVARES V.I., VALENZUELA M.K., JIMENEZ A.G. \& CACCIUTTOLO J.C. Pautas de Evaluación de las Habilidades Motrices Básicas de botear un balón, rodar hacia adelante y hacer rodar un balón en niños y niñas de 6 y 7 años de edad. Mot.Hum. 13(2) : 74-83; 2012.

\section{INTRODUCCIÓN}

Los procesos evolutivos de la motricidad, especialmente en la infancia, transcurren de manera rápida y compleja; y es en esta etapa del ciclo vital donde ocurren la mayor cantidad de cambios desde el punto de vista de la motricidad natural, base para el aprendizaje de habilidades mas complejas como las deportivas, artísticas o laborales, como también para desenvolverse en las actividades de la vida diaria de toda persona. Es así como en esta etapa adquieren prioridad aquellas formas naturales de movimiento que son propias de la infancia, las habilidades motrices básicas, las cuales poseen su periodo crítico de desarrollo, y se constituyen como la base para movimientos mas especializados. Estas habilidades otorgan la capacidad de desplazarse, equilibrarse y manipular objetos de manera natural, voluntaria, autónoma y eficiente $(1,2,3,4)$.

En este contexto, el conocer y comprender desde una perspectiva educativa como transcurren los procesos evolutivos de la motricidad en la infancia, concretamente desde la educación física, cobra suma relevancia (5), debido a que posibilita a los docentes plantear lineamientos didácticos que permitirán orientar los procesos de enseñanza y establecer líneas de acción concretas a través de una mediación pedagógica oportuna y sistemática en el tiempo, posibilitando vivencias gratificantes $\mathrm{y}$ exitosas, y de esta manera niños y niñas logren integrarse de manera positiva en y con su entorno.

Lo anterior plantea como desafío a los profesionales de la educación física el deber conocer el contexto en el cual se desenvuelve para implementar las metodologías adecuadas $(6,7)$, para ello, es prioritario conocer el estado inicial de sus estudiantes, fundamentalmente sus características motrices, para establecer líneas de acción y planificar los recursos de enseñanza en beneficio de la mejora de este nivel inicial. $(8,9)$. Es importante constatar característica en el desarrollo motriz del infante que pueda ser considerada como anormal, para poder establecer estrategias de soluciones pertinentes y coherentes a la etapa en que se encuentran (10).
La evaluación de la motricidad en la infancia, específicamente de las habilidades motrices básicas, colabora en identificar cuales son aquellas que están deficitarias, para que los profesores implementen decisiones pertinentes para proponer vías de retroalimentación a dichos elementos deficitarios (11).

En relación a los procesos evaluativos ligados al desarrollo motriz en la infancia, se han investigado estos procesos desde perspectivas diferentes $(1,12$, $13,14,15,16,17,18)$, de las cuales emanan una variedad de baterías o pruebas que proponen diversas modalidades de procedimientos y aspectos a evaluar, y recorren un amplio espectro de propuestas que van desde las que evalúan aspectos puramente motrices $(1,2,16,18)$, a aquellas que evaluar otras dimensiones de la persona por medio de su motricidad (15).

Estas evaluaciones deben ser realizadas en la etapa de la infancia, fundamentalmente debido a que alrededor de los 6 años de edad es cuando niños y niñas poseen el máximo potencial de desarrollo para lograr la etapa madura de la mayoría de las habilidades motrices básicas (3). La infancia es la etapa del ciclo vital en la cual donde se cimientan las bases del desarrollo motriz, constituyéndose en los pilares fundamentales para los aprendizajes motrices posteriores, especializados y mas complejos, tanto para la vida cotidiana, el deporte, la recreación, lo artístico y lo laboral.

Desde una perspectiva neurológica, generalmente alrededor de los cuatro o cinco años es cuando se logran con mayor velocidad los procesos de aprendizajes, debido fundamentalmente a la organización de los sistemas sensoriales y motrices, es decir, el sistema sensorial se encuentra en óptimas condiciones madurativas (19).

A estas edades, se concretan en gran medida los procesos madurativos del sistema nervioso central, sus estructuras constituyentes se encuentran bien conformadas y desarrolladas. El cerebro, gracias al gran potencial de establecer diversos contactos neuronales, posee gran plasticidad, lo que facilita la posibilidad cierta de aprender, $(20,21)$. Entre el 
GAMBOA J.R., ACEVEDO G.C., CANALA-ECHEVERRIA T.S., OLIVARES V.I., VALENZUELA M.K., JIMENEZ A.G. \& CACCIUTTOLO J.C. Pautas de Evaluación de las Habilidades Motrices Básicas de botear un balón, rodar hacia adelante y hacer rodar un balón en niños y niñas de 6 y 7 años de edad. Mot.Hum. 13(2) : 74-83; 2012.

cuarto y octavo año de vida aproximadamente, el número de sinapsis es muy superior que las de un adulto, debido a la ya señalada plasticidad que posee el cerebro (21).

Conjuntamente a lo anterior, el mayor desarrollo perceptivo motriz, procesos que inciden y poseen un rol esencial en evolución de la motricidad, tiene lugar entre el tercer y séptimo año de vida, consideración que también justifica lo ya planteado en relación a que la infancia es la etapa crítica para el desarrollo de la motricidad (22).

Sin perjuicio de lo anterior, existe otro elemento esencial en el posible logro del patrón maduro de estas habilidades, el ambiente como factor que incide en el desarrollo motriz de las personas. Se plantean que el dejar el desarrollo motriz en mano sólo de los procesos de maduración, las personas no lograrán el desarrollo óptimo de las habilidades. El ambiente se conjuga con los procesos madurativos y potencialidades motrices personales para crear las condiciones necesarias para los diferentes logros en lo motriz $(2,5,23,24,25)$.

Al realizar una relación entre ambos factores, ambiente y maduración, se puede señalar que la fijación de los circuitos neuronales, y la creación de nuevos, se da gracias a la experiencia tanto en actividades motrices como en las cognitivas (21).

Esta última idea señalada cobra relevancia debido a que generalmente se señala la incidencia de los efectos de los procesos neurológicos en el desarrollo de las personas, sin embargo, se debe destacar que el ambiente también colabora en los procesos de maduración del sistema nervioso. El ambiente es esencial en el desarrollo neurológico, tanto para la maduración y desarrollo de estos sistemas, como para el mantenimiento de las funciones del mismo (26).

$\mathrm{Si}$ en estas edades se presentan las anteriores condiciones óptimas de desarrollo, niños y niñas a las edades de seis, siete u ocho años ya debiesen tener adquiridos y desarrollados sus movimientos de carácter natural e inherentes a su especie, las habilidades motrices básicas, y en ese contexto, estar capacitados para realizar diversas tareas motrices que colabore con el logro de la madurez de ellas.

En este contexto, se ha diseñado y validado ante el juicio de experto y de manera empírica en una realidad educativa, un conjunto de pautas que sitúan el énfasis de su evaluación en el cómo se realiza la habilidad motriz básica, sin perjuicio de los enormes aportes entregados por los diversos protocolos anteriormente señalados, $\mathrm{u}$ otros que evalúen estos aspectos en la infancia.

Es esencial para la realidad nacional, y fundamentalmente para la realidad escolar, contar con procedimientos que evalúen no sólo la presencia o ausencia de la habilidad motriz básica, que también es un elemento fundamental a considerar en edades tempranas, sino que además evalúen el cómo se realiza, evaluar la calidad de ejecución, es decir, el nivel de desarrollo logrado por el niño o niña en relación al patrón maduro de dicha habilidad. Entendiéndose por patrón maduro el modelo teórico de referencia de cómo se realiza una habilidad motriz básica de manera eficiente. $(23,27,28,29)$.

El obtener esta información relevante de cómo se ejecuta la habilidad y cada uno de sus elementos constituyentes, permite a los y las docentes tomar decisiones didácticas, y con ello, intencionar la tarea en aquellos elementos de la habilidad que se encuentran deficitarios en beneficio del desarrollo de dicha habilidad, y por tanto de la persona.

En la década de los 80 ya realizaban este planteamiento, y se señalaba que la mayoría de los instrumentos a la fecha de su publicación, ponían el énfasis de la evaluación en la cantidad más que en la calidad de ejecución de las habilidades motrices básicas, lo que no da la posibilidad cierta de constatar por parte del docente las debilidades y fortalezas presentes en la ejecución (1).

En concreto, las pautas propuestas permiten evaluar la calidad de ejecución de tres habilidades motrices básicas: botear un balón con mano dominante, rodar hacia delante y hacer rodar un balón con mano 
GAMBOA J.R., ACEVEDO G.C., CANALA-ECHEVERRIA T.S., OLIVARES V.I., VALENZUELA M.K., JIMENEZ A.G. \& CACCIUTTOLO J.C. Pautas de Evaluación de las Habilidades Motrices Básicas de botear un balón, rodar hacia adelante y hacer rodar un balón en niños y niñas de 6 y 7 años de edad. Mot.Hum. 13(2) : 74-83; 2012.

dominante. Es importante mencionar que cada una estas habilidades seleccionadas han sido escogidas por ser consideradas, junto a otras habilidades, en las más presentes en los juegos de la etapa de la infancia, y constituyen la base para el aprendizaje de habilidades más complejas $(3,27,30)$. Conjuntamente a lo anterior, son constitutivas de diversos protocolos de evaluación (23).

\section{MATERIAL Y MÉTODO}

La presente investigación de carácter cuantitativo, ha utilizado una muestra de carácter no probabilística intencionada compuesta por 100 estudiantes de 6 y 7 años de edad de la ciudad de Quilpué que asisten al establecimientos educacional Colegio Aconcagua, 50 niños y 50 niñas.

Para el diseño de las pautas de evaluación de las tres habilidades motrices básicas se ha seguido el siguiente procedimiento:

1. Revisión material bibliográfico de habilidades motrices básicas, su descripción y procesos evolutivas $(1,3,4,27)$.

2. Revisión material bibliográfico de protocolos de evaluación de habilidades motrices básicas, fundamentalmente en la etapa de la infancia (1, 3, $12,13,14,15,16,17,29)$.

3. Selección de bibliografía base a utilizar, fundamentalmente aquellas que son coherentes con los propósitos del presente estudio. Se ha seleccionado principalmente los trabajos de Mc Clenaghan y Gallahue; Gallahue y Ozmun; Gamboa y colaboradores $(1,3,29)$, sin dejar de considerar los valiosos aportes entregados por otros autores.

4. Selección de las habilidades motrices básicas partes de la investigación. Se ha decidido por habilidades propuestas esencialmente por Gallahue y Ozmun (3).

5. Se identifican los elementos más relevantes y observables de cada una de las habilidades según su patrón maduro. Rasgos esenciales de cada habilidad.
6. Aquellos elementos relevantes se transforman en indicadores observables.

7. Capacitación de evaluadores que aplicarán las pautas a la muestra parte del estudio. Se realizan tres pruebas pilotos, se analizan videos e imágenes, y se comparten reflexiones de este proceso para aunar criterios de observación.

Para la validación de contenido de las pautas de evaluación, se someten al juicio de expertos. Se le solicita den su juicio sobre la claridad, pertinencia y coherencia de los distintos aspectos que componen las pautas, y posibles sugerencias para la mejora de las mismas. Se aplica el coeficiente de correlación de Spearman, con un nivel de significancia $=0,05 \mathrm{y}$ con un $95 \%$ de confianza respecto a si existen diferencias significativas a partir de las opiniones expresadas por los expertos.

Para obtener el grado de fiabilidad, es decir, para identificar la confiabilidad, consistencia y objetividad interobservador, se realizaron las evaluaciones en tres oportunidades distintas, pero bajo las mismas condiciones. Lo anterior se realiza través de los siguientes pasos:

1. La primera y segunda evaluación se realizan por los evaluadores 1 y 2 , de modo de comprobar la correlación que existe entre ellos en un mismo momento de aplicación. Para ello se aplica el confiabilidad: coeficiente de correlación interobservador Índice KAPPA. Conjuntamente a lo anterior, se calcula la consistencia de los instrumentos, es decir, comprobar la correlación de puntuaciones en momentos diferentes de aplicación a través del índice ANOVA, ccon nivel de significancia 0,05 y $95 \%$ de confianza.

2. la tercera evaluación se realiza por los evaluadores 2 y 3 , con la finalidad de obtener hallazgos fidedignos que evidencien que los instrumentos poseen altos grados de objetividad, es decir, permitirán ser aplicados por distintos evaluadores. Es decir, que se obtengan puntuaciones similares entre evaluadores $1-2$ y $2-3$. Para este caso, se aplica el, Test de comparaciones múltiples de Tukey. 


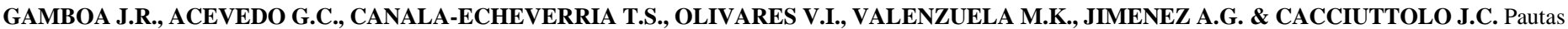

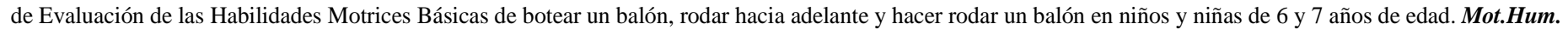
13(2) : 74-83; 2012.

Para obtener los valores anteriormente señalados, se realizaron a través del paquete estadístico software SPSS:

\section{RESULTADOS}

En primer lugar, para el análisis del nivel de concordancia entre los tres jueces validadores del contenido se aplica la prueba estadística coeficiente de correlación de Spearman. De este análisis, no ha sido posible obtener dicho coeficiente, debido a que en las opiniones del experto 2 existe una constante,

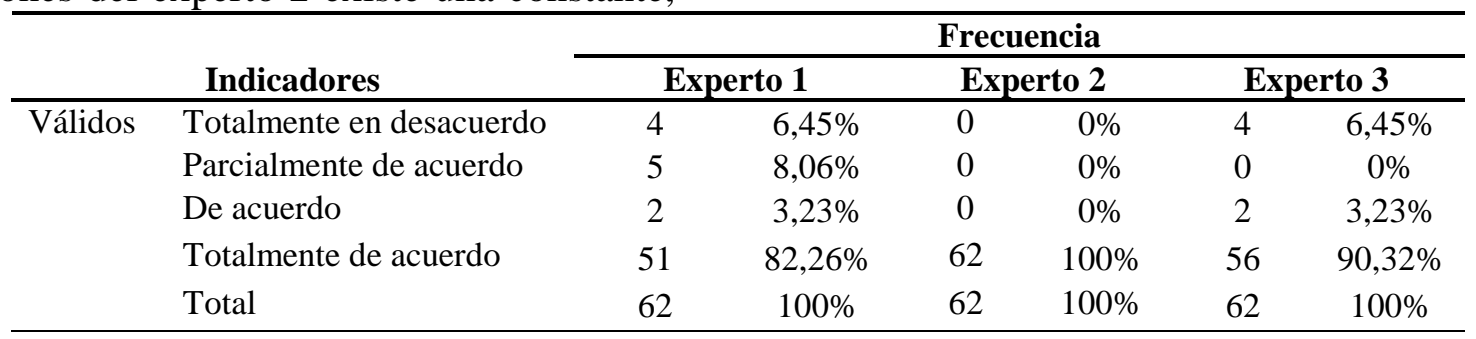

Tabla I: Distribución de frecuencia. Expoertos validación de contenido.

Se puede observar en la tabla $\mathrm{n}^{\circ} \mathrm{I}$, que los expertos coinciden en la categoría "totalmente de acuerdo" en 51 casos de un total de 62 posibles, lo que representa el $82,26 \%$ del total. Es importante relevar, que del total de esas 51 respuestas coincidentes, el $100 \%$ corresponde en las mismas preguntas, lo que permite señalar que sólo el $17,74 \%$ de las respuestas no son coincidentes.

En segundo lugar, para obtener el coeficiente de correlación interobservador, es decir, grado de concordancia entre las puntuaciones de dos o mas el $100 \%$ de ellas son "totalmente de acuerdo".

Sin perjuicio de lo anterior, a través de la estadística descriptiva, análisis de frecuencia expresada en porcentajes realizado previamente al coeficiente Spearman, Tabla I, se puede inferir que el grado de concordancia es alto.

evaluadores en un mismo momento, se aploicó el índice Kappa, de modo de determinar el grado de concordancia al interior de las evaluaciones del día 1 , día 2 y día 3.

De lo señalado en la Tabla II, se puede señalar que en general en todos los indicadores que componen la habilidad motora básica de botear un balón con mano dominante, el nivel de acuerdo respecto al índice Kappa, para el día 1 y 2 son considerado como muy bueno, debido a que se han obtenido valores muy cercanos a " 1 ". Y para el día 3, son considerados como buenos.

\begin{tabular}{|c|c|c|c|}
\hline \multirow{2}{*}{$\begin{array}{l}\text { Indicadores habilidad motriz básica: } \\
\text { Botear un balón con mano dominante }\end{array}$} & \multicolumn{3}{|c|}{ Indice KAPPA } \\
\hline & Día 1 & Día 2 & Día 3 \\
\hline $\begin{array}{l}\text { 1. El pie opuesto a la mano que sostiene el balón está desplazado } \\
\text { hacia delante. }\end{array}$ & 0,921 & 0,987 & 0,834 \\
\hline 2. El tronco se mantiene levemente inclinado hacia delante & 0,953 & 0,973 & 0,844 \\
\hline $\begin{array}{l}\text { 3. Al botear el balón, la mano se adapta a la forma de éste, y el } \\
\text { contracto se realiza a la altura de la cintura. }\end{array}$ & 0,939 & 0,980 & 0,834 \\
\hline $\begin{array}{l}\text { 4. La acción se realiza por movimientos sucesivos de hombro, codo } \\
\text { y muñeca, a lo menos tres veces. }\end{array}$ & 0,905 & 1,000 & 0,890 \\
\hline 5. Dirige su vista al frente & 0,905 &. $\mathrm{a}$ & 0,890 \\
\hline 6. Lo hace en ausencia de sincinesias. & 0,973 & 0,987 & 0,835 \\
\hline
\end{tabular}

Tabla II: Índice Kappa. Botear un balón con mano dominante. 


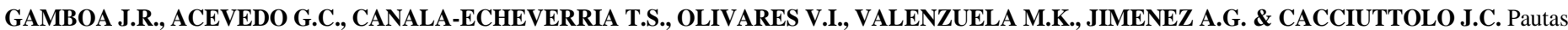

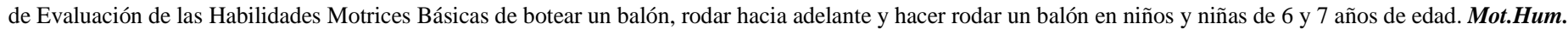
13(2) : 74-83; 2012.

Para el indicador $n^{\circ} 5$, día 2 , su resultado no se pudo calcular debido a que existe una constante en el juez 2. Sin embargo, a partir del resultado de la estadística descriptiva, se observó que mientras el $100 \%$ de los resultados de la opinión del juez 1 es "no aplica", este porcentaje también equivale al $100 \%$ al realizar la misma pregunta al juez 2, por tanto el porcentaje de coincidencias se situó en el
$100 \%$, de lo que se puede inferir que estadísticamente el acuerdo fue muy bueno.

Para la habilidad de rodar adelante, Tabla III, se puede señalar que en general los indices Kappa para los tres días permiten clasificar los acuerdos como bueno, valores entre ,800 y ,900; y como acuerdos muy buenos, estos últimos debido a que los valores son muy cercanos a "1".

\begin{tabular}{|c|c|c|c|}
\hline \multirow{2}{*}{$\begin{array}{l}\text { Indicadores habilidad motriz básica: } \\
\text { Rodar adelante }\end{array}$} & \multicolumn{3}{|c|}{ Indice KAPPA } \\
\hline & Día 1 & Día 2 & Día 3 \\
\hline $\begin{array}{l}\text { 1. Las extremidades superiores se desplazan desde el costado del cuerpo } \\
\text { hacia adelante, al mismo tiempo que inclina el tronco para preparar la } \\
\text { ejecución. }\end{array}$ & 0,807 & 0,947 & 0,848 \\
\hline 2. Las manos se apoyan de manera simultánea. & 0,984 & 0,965 & 0,881 \\
\hline $\begin{array}{l}\text { 3. Durante el momento de rodar, mantiene el cuerpo ovillado con apoyo } \\
\text { sucesivo de nuca, dorso y coxis. }\end{array}$ & 0,979 & 1,000 & 0,869 \\
\hline $\begin{array}{l}\text { 4. Al finalizar vuelve a la posición de pie en colaboración de las } \\
\text { extremidades superiores que se dirigen hacia adelante-arriba, controlando el } \\
\text { equilibrio. }\end{array}$ & 0,979 & 0,820 & 0,881 \\
\hline 5. Lo hace en ausencia de sincinesias. & 0,852 & 0,862 & 0,834 \\
\hline
\end{tabular}

Tabla III: Índice Kappa. Rodar adelante

Para la habilidad de hacer rodar un balor con mano dominante, Tabla IV, en general el nivel de acuerdo respecto al índice Kappa, para los días 1 y 2 son considerado como muy bueno, debido a que son valores muy cercanos a "1". Y para el día 3, son considerados como buenos, rangos entre $0,800 \mathrm{y}$ 0,900 .

\begin{tabular}{lccc}
\hline \multicolumn{1}{c}{$\begin{array}{c}\text { Indicadores habilidad motriz básica: } \\
\text { Hacer rodar un balón con mano dominante }\end{array}$} & \multicolumn{1}{c}{ Indice KAPPA } \\
\cline { 2 - 4 } & Día 1 & Día 2 & Día 3 \\
\hline $\begin{array}{l}\text { 1. Al hacer rodar el balón. Se ubica con el tronco inclinado hacia delante, con } \\
\text { flexión de rodillas y el pie contrario a la mano que hace rodar el balón, se } \\
\text { encuentra adelantado. }\end{array}$ & 0,981 & 0,978 & 0,886 \\
$\begin{array}{l}\text { 2. La extremidad de la mano que hace rodar el balón, se desplaza desde atrás } \\
\text { hacia adelante para darle impulso a este. }\end{array}$ & 0,921 & 0,974 & 0,852 \\
$\begin{array}{l}\text { 3. Desplaza el peso del cuerpo desde atrás hacia adelante en el momento de } \\
\text { hacer rodar el balón. }\end{array}$ & 0,951 & 0,987 & 0,645 \\
$\begin{array}{l}\text { 4. En la liberación del balón, éste rueda por la mano que lo sostiene al mismo } \\
\text { tiempo que es impulsado hacia delante. }\end{array}$ & 0,971 & 0,980 & 0,854 \\
$\begin{array}{l}\text { 5. Al momento de hacer rodar el balón, aproxima el tronco al suelo. } \\
\text { 6. Lo hace en ausencia de sincinesias }\end{array}$ & 0,905 & 0,942 & 0,854 \\
\hline
\end{tabular}

Tabla IV: Índice Kappa. Hacer rodar un balón con mano dominante

En tercer lugar, respecto a la consistencia de los instrumentos de evaluación, es decir, responde a que si al aplicar la evaluación en días distintos, a una misma muestra, se obtienen resultados similares, de este modo se cautela que no existan diferencias estadísticamente significativas, lo cual es un elemento importante de la fiabilidad, se aploicó el índice ANOVA. 
GAMBOA J.R., ACEVEDO G.C., CANALA-ECHEVERRIA T.S., OLIVARES V.I., VALENZUELA M.K., JIMENEZ A.G. \& CACCIUTTOLO J.C. Pautas de Evaluación de las Habilidades Motrices Básicas de botear un balón, rodar hacia adelante y hacer rodar un balón en niños y niñas de 6 y 7 años de edad. Mot.Hum. $13(2): 74-83 ; 2012$.

\begin{tabular}{lc}
\hline \multicolumn{1}{c}{ Habilidades Motrices Básicas } & $\begin{array}{c}\text { Indice } \\
\text { ANOVA }\end{array}$ \\
\hline $\begin{array}{l}\text { Hacer rodar un balón con mano } \\
\text { dominante }\end{array}$ & 0,286 \\
$\begin{array}{l}\text { Botear un balón con mano } \\
\text { dominante }\end{array}$ & 0,221 \\
Rodar adelante. & 0,091 \\
\hline
\end{tabular}

Tabla V: Índice ANOVA

En la Tabla V, se puede observar que en las habilidad de hacer rodar un balón con mano dominante, botear un balón con mano dominante y

rodar adelante, el valor de probabilidad $\mathrm{p}=0,286$; $\mathrm{p}=0,221 ; \mathrm{y} \mathrm{p}=0,091$ respectivamente, es mayor para los tres casos respecto a 0,05 , lo que significa que las pruebas son fuertemente significativa al $5 \%$.

\begin{tabular}{|c|c|c|c|c|}
\hline (I) Día & (J) Día & $\begin{array}{c}\text { Hacer rodar balón } \\
\text { Sig. }\end{array}$ & $\begin{array}{c}\text { Tukey } \\
\text { Botear un balón } \\
\text { Sig. } \\
\end{array}$ & $\begin{array}{c}\text { Rodar adelante } \\
\text { Sig. }\end{array}$ \\
\hline \multirow[t]{2}{*}{ HSD de Tukey 1} & 2 & 0,704 & 0,809 & 0,851 \\
\hline & 3 & 0,254 & 0,516 & 0,259 \\
\hline \multirow[t]{2}{*}{ HSD de Tukey 2} & 1 & 0,704 & 0,809 & 0,851 \\
\hline & 3 & 0,713 & 0,200 & 0,088 \\
\hline \multirow[t]{2}{*}{ HSD de Tukey 3} & 1 & 0,254 & 0,516 & 0,259 \\
\hline & 2 & 0,713 & 0,200 & 0,088 \\
\hline
\end{tabular}

Tabla VI: Test de comparaciones múltiples de Tukey

Los resultados de las comparaciones múltiples según el método de Tukey, señalan que no hay diferencia significativa entre los puntajes medios entre los días de aplicación para las tres habilidades motrices básicas, debido a que el valor $\mathrm{p}$ de significancia es mayor a 0,05 . Lo anterior permite señalar que no existen diferencias estadísticamente significativas entre las puntuaciones otorgadas por los distintos jueces evaluadores en los distintos días.
Por lo tanto, los puntajes de los jueces son similares en la aplicación en los distintos días.

En cuarto lugar, para determinar el grado de objetividad interobservador, se ha aplicado el test de comparaciones multiples de Tukey, Tabla VI. Lo anterior permite identificar si hay discrepancias entre los jueces 1 y 2 , con respecto a los jueces 3 y 4, es decir, responde a que al aplicar las evaluaciones por evaluadores distintos a una misma muestra, en días diferentes, se dan resultados similares, otro elemento importante en la fiabilidad de un instrumento. 


\begin{tabular}{|c|c|}
\hline $\begin{array}{l}\text { Habilidad motriz } \\
\text { básica }\end{array}$ & Indicadores por habilidad motriz básica \\
\hline $\begin{array}{l}\text { Hacer rodar un balón } \\
\text { con mano dominante }\end{array}$ & $\begin{array}{l}\text { 1. Al hacer rodar el balón, se ubica con el tronco inclinado hacia delante, con } \\
\text { flexión de rodillas y el pie contrario a la mano que hace rodar el balón, se } \\
\text { encuestra adelantado } \\
\text { 2. La extremidad de la mano que hace rodar el balón, se desplaza desde atrás } \\
\text { hacia adelante para darle impulso a este. } \\
\text { 3. Desplaza el peso del cuerpo desde atrás hacia adelante en el } \\
\text { momento de hacer rodar el balón. } \\
\text { 4. En la liberación del balón, este rueda por la mano que lo sostiene al } \\
\text { mismo tiempo que es impulsado hacia delante } \\
\text { 5. Al momento de hacer rodar el balón, aproxima el tronco al suelo. } \\
\text { 6. Lo hace en ausencia de sincinesias. }\end{array}$ \\
\hline $\begin{array}{l}\text { Botear un balón con } \\
\text { mano dominante }\end{array}$ & $\begin{array}{l}\text { 1. El pie opuesto pie a la mano que sostiene el balón está desplazado hacia } \\
\text { adelante. } \\
\text { 2. El tronco se mantiene levemente inclinado hacia delante. } \\
\text { 3. Al botear el balón, la mano se adapta a la forma de este, y el contacto se } \\
\text { realiza a la altura de la cintura. } \\
\text { 4. La acción se realiza por movimientos sucesivos de hombro codo y } \\
\text { muñeca, a lo menos tres veces. } \\
\text { 5. Dirige su vista al frente. } \\
\text { 6. Lo hace en ausencia de sincinesias. }\end{array}$ \\
\hline Rodar adelante. & $\begin{array}{l}\text { 1. Las extremidades superiores se desplazan desde el costado del cuerpo } \\
\text { hacia adelante, al mismo tiempo que inclina el tronco para preparar la } \\
\text { ejecución. } \\
\text { 2. Las manos se apoyan de manera simultánea. } \\
\text { 3. Durante el momento de rodar, mantiene el cuerpo ovillado con apoyos } \\
\text { sucesivos de nuca, dorso y coxis. } \\
\text { 4. Al finalizar vuelve a la posición de pie en colaboración de las } \\
\text { extremidades superiores que se dirigen adelante arriba, controlando el } \\
\text { equilibrio. } \\
\text { 5. Lo hace en ausencia de sincinesias. }\end{array}$ \\
\hline
\end{tabular}

Tabla VII: Habilidades motrices básicas y sus respectivos indicadores.

\section{DISCUSIÓN}

El primer término, es importante relevar que se ha logrado diseñar y validar un conjunto de pautas de evaluación de la calidad de ejecución de tres habilidades motrices básicas: hacer rodar un balón con mano dominante, rodar adelante y botear un balón con mano dominante.

En segundo término, tal y como se planea en la teoría, es fundamental disponer de instrumentos de evaluación de habilidades que centren su atención en la calidad de ejecución, mas que la presencia o ausencia de ellas. Esto permitirá a los y las docentes a planear líneas de acción e intencionar la tarea de modo de favorecer el desarrollo de aquellos elementos deficitarios de la habilidad en vista a lograr el patrón maduro de dicha habilidad $(1,2,3)$.

Finalmente, tanto para la validación del contenido, la confiabilidad, la consistencia y la objetividad interobservador de las pautas de evaluación, se han logrado altos grados de acuerdo para el primer caso, y altos índices de concordancia para los otros tres. Lo cual permite determinar que el proceso de validación ha culminado exitosamente. 


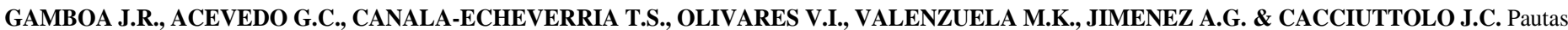

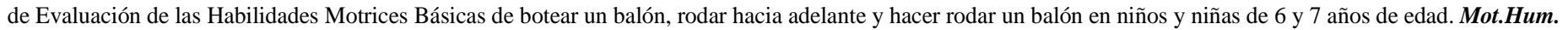
13(2) : 74-83; 2012.

\section{REFERENCIAS BIBLIOGRAFICAS}

1. Mc Clenaghan, B. \& Gallahue, D. Movimientos fundamentales: su desarrollo y rehabilitación. Buenos Aires: Medica Panamericana S.A. 1985.

2. Gallahue, D. \& Ozmun, J. Motor development. children, infants, adolescent, adults. $\left(2^{\mathrm{a}}\right.$ ed $)$. Indiana: Brown and Benchmark. 1995.

3. Gallahue, D. \& Ozmun, J. Understanding motor development. infants, children, adolescents, adults. (6o ed). Indiana: Mc Graw Hill. 2006.

4. Gamboa, R. Desarrollo motriz: habilidad motriz básica y patrón de movimiento. España: Editorial Académica Española. (2012).

5. Gamboa, R. Diferencias de género en la ejecución de habilidades motoras básicas en pre escolares chilenos. En Revista Motricidad Humana 2010: 10 (2): 87-94.

6. Giralde, M. Brizzi, H. \& Madueño, J. Didáctica de la educación física. Buenos Aires: Facula R.S.L. 1998.

7. Gobierno de Chile. Marco para la buena enseñanza. Santiago: Ministerio de Educación. 2003.

8. González, C. \& Lleixá, T. Didáctica de la Educación Física. Barcelona: Graò. 2010.

9. Ruiz, L. Graupera, J. Competencia motriz y género entre los escolares españoles. En Revista Internacional de Medicina y Ciencias de la Actividad Física y del Deporte 2003: 3 (10); 101-111.

10. Hormiga, C. Camargo, D. Orozco, L. Reproducibilidad y validez convergente de la escala abreviada del desarrollo y una traducción al español del instrumento Neurosensory Motor Development Assessment. En Biomedica 2008: 28 (3); 32720.

11. Valentíni, N. Lopez, M. Villwock, G. Karine, R. Coiro, B. \& Abaide, M. Teste de desenvolvimento motor grosso: validade e consistencia interna para una poblaçâo gaúcha. En Revista Brasileira de Cineantropometria \& Desempenho Humano 2008: 10 (4); 399-404.

12. Trujillo, H. Caro, M. Aranda, R. Latorre, R. Valcke, S. \& Orellana, A. Validación quinta versión BAPERMOC. Serie de Estudios, 232. 1994.

13. Heussler, I. \& Marchant, T. Tepsi: test de desarrollo psicomotor de 2 a 5 años. ( $5^{\mathrm{a}}$ ed). Santiago: Pontificia Universidad Católica de Chile. 1994.

14. Franterburg, W. Denver development screeting test
(D.D.S.T.). USA: University of Colorado Medical Center. 1975.

15. Da Fonseca, V. Manual de observación psicomotriz. ( $3^{\mathrm{a}}$ ed). Barcelona: Inde. 2008.

16. Gallahue, D. Developmental physical education for today children. ( $2^{\mathrm{a}}$ ed). Indiana: Brown and Benchmark. 1987.

17. Sugden, D. \& Henderson, S. Movement assessment battery for children. London: Psichological Corporation. 1992.

18. Vargas, C. Tesis Doctoral. Pauta de observación de patrones motores en niños de 4 a 5 años: diseño, validación, observación de desarrollo motor y propuesta de estimulación. Universidad de Valladolid. Facultad de Educación y Trabajo Social. Departamento de Pedagogía. Valladolid. 2004.

19. Castañer, M. \& Camerino, O. La educación física en la enseñanza primaria. Barcelona: Inde. 2001.

20. Cerda, J. Polanco, A. \& Rojas, P. El niño entre cuatro y cinco años: características sobre su desarrollo socioemocional, psicomotriz y cognitivo - lingüístico. En Educación 2002: 28 (1); $169-183$.

21. Rigal, R. Educación motriz y educación psicomotriz en preescolar y primaria. Barcelona: Inde. 2006.

22. Papalia, D. Wendkos, S. Duskin, R. Psicología del desarrollo: de la infancia a la adolescencia. ( $9^{\circ}$ ed). México: Mcgraw-Hill. 2005.

23. Gamboa, R. Diferencias de género en la ejecución de habilidades motoras básicas en pre escolares chilenos. En Revista Motricidad Humana (2010): 10 (2); 87-94.

24. Hui, J. y Wang, T. A study on gross motor skills of preschool children. En Journal of Research in Childhood Education 2004: 19 (1); 32-12.

25. Shala, M. Assessing gross motor skills of Kosovar preschool children. En Early Child Development and Care 2009: 179 (7); 969-976.

26. Ajuriaguerra, J. Manual de psiquiatría infantil. Barcelona: Masson. 1977.

27, Wickstrom, R. Patrones motores básicos. Madrid: Alianza Deportes. 1990.

28. Ruiz, L. Deporte y aprendizaje. Procesos de adquisición y desarrollo de habilidades. Madrid: Visor. 1994.

29. Gamboa, R. Jiménez, G. González, L. Cacciuttolo, C. \& Poblete, C. Pautas de evaluación de seis habilidades motrices básicas en niños y niñas de 5 y 6 años de edad. En Revista 
Revista Motricidad Humana / Julio - Diciembre, Edición 13(2)

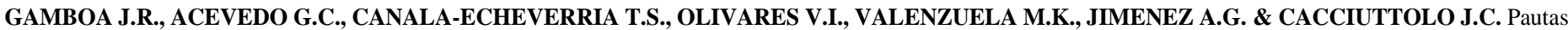

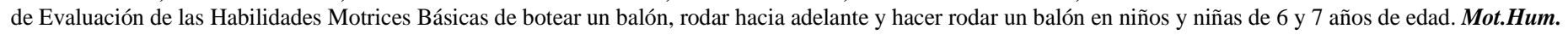
13(2) : 74-83; 2012.

Motricidad Humana 2012: 13 (1): 20-27.

30. Ruiz, L. Desarrollo motor y actividades físicas. Madrid: Gymnos. 1987.

\section{Dirigir Correspondencia a:}

Dr. Rodrigo Gamboa Jiménez. rodrigo.gamboa@ucv.cl

Av. El Bosque 1290. Viña del Mar. Chile. 56-32-2274304.

RECIBIDO: 10-10-2012

ACEPTADO: 16-11-2012 